Disclosures M. de Miquel: None. L. Aja: None. S. Aixut: None. R. Barranco: None. O. Chirife: None. P. Cardona: None. H. Quesada: None. B. Lara: None. A. Paipa: None.

\section{E-128 DISRUPTING CLOT ADHESION TO THE VESSEL WALL IS AN ESSENTIAL STEP OF DIRECT THROMBUS ASPIRATION}

L Miskolczi*. Holy Cross Hospital, Ft. Lauderdale, FL

10.1136/neurintsurg-2019-SNIS.203

Introduction There are some misconceptions and misunderstandings regarding various implementations of what is generally called A Direct Aspiration First Pass Technique (ADAPT). Variants of the technique may result in significantly different results. We propose a modification of the ADAPT technique that promises faster procedures because of higher rates of single-pass recanalization.

Materials and methods Three years (2016-2018) of prospectively collected data on 147 consecutive thrombectomies by a single operator were reviewed retrospectively that included 68 ADAPT cases. The ratio of stent retriever cases versus ADAPT cases were compared. During this 3 -year period a new catheter handling technique has been implemented, that intends to mobilize the clot before retrieval.

Video animations suggest transcatheter aspiration of a Jellolike substance via the tubing into a canister. In real life that rarely happens. Instead, the goal is to have the embolus wedged, corked into the distal tip of the catheter with the help of vacuum, then to physically remove it along with the catheter, preferably in one piece. We recognized that vacuum alone is frequently insufficient to grab, mobilize and remove the embolus in one piece. Some extra effort, or force, is needed to disrupt the adhesion of the clot to the vessel wall it is wedged in. The pulling force is limited by the vacuum lock between the catheter tip and the embolus. However, a pushing force can be stronger, achieving two goals simultaneously: disrupt the clot adhesion, and help stronger corking of the embolus into the catheter tip where the vacuum will be able to hold it stronger during removal.

The guide sheath is advanced as high as possible to provide better support. The aspiration catheter is then advanced right in front of the embolus and vacuum is turned on. The catheter is then pushed forward, beyond the original position of the embolus, during continuous aspiration. If the catheter moves forward with ease, the embolus is almost certainly mobilized and locked. The catheter can be immediately removed.

The success or failure of this forward-push technique was correlated with ADAPT ONLY success versus cross-over to stent retriever, based on our fluoroscopic videos.

Results During the 3-year period the ratio of ADAPT ONLY cases grew from $35 \%$ to $61 \%$. The ratio of ADAPT attempts, eventually crossed over to stent retriever, decreased from $46 \%$ to $18 \%$. Forward-push technique was used in 4,13 and 24 cases in the 3 subsequent years. None of them (0 of 41) required crossing over to stent retriever. During the same 3 years forward-push technique was not used or failed in 26, 20 and 20 cases respectively, of them 17 (65\%), $12(60 \%)$ and $10(50 \%)$ required crossing over to stent retrievers.

Conclusion Adhesion disruption using the forward-push technique helps to mobilize the embolus and to prepare it for a more efficient, successful removal in a single piece. Further testing, including bench-top evaluation will be needed to optimize the technique.

Disclosures L. Miskolczi: None.

\section{E-129 THROMBECTOMY IN DISTAL VESSEL OCCLUSION: MCA M2-3 JUNCTION AND BEYOND}

B Donegan*, J Loeb, C Martin, W Holloway, J Halpin, N Akhtar. Saint Luke's Marion Bloch Neuroscience Institute, Kansas City, MO

10.1136/neurintsurg-2019-SNIS.204

Introduction/Purpose Since 2012, our team of neurointerventional physicians have performed over 600 intracranial arterial thrombectomies with stent-retrievers. Thrombectomy in the ICA and M1 segments has been shown in randomized trials to reduce permanent disability and is now the standard of care. The time window has continued to expand. However, less is known about outcomes for thrombectomy performed in distal branches, such as near the M2-3 junction or within the M3 segments. Current guidelines state that thrombectomy in these locations may be performed in carefully selected patients, however data is limited.

Materials and methods A retrospective review of our stroke database from 2012-2018 was undertaken to find cases of MCA interventions involving the distal third of the M2 insular segment or the M3 opercular segment. Isolated ICA, M1 and proximal M2 branch occlusions, as well as proximal occlusions that resulted in distal emboli post-thrombectomy, were excluded. Patients were selected for intervention based on noncontrast CT head, CTA, CT perfusion results; onset time; and clinical exam. Distal vessel thrombectomies were performed with the use of a stent-retriever device. Pre-intervention scans were compared to post-intervention scans to evaluate degree of infarct. The baseline and discharge National Institute of Health Stroke Scale, and the modified Rankin Scale at 90 days were used to evaluate the degree of residual deficits.

Results A total of 43 patients met the inclusion criteria. Mean age was 69.4 (IQR 60.5-82). 23 (53.5\%) were female. Thrombus was categorized as being located completely within the M3 segment in 21 cases (48.8\%) and spanning the M2M3 junction in 22 cases. 6 patients (14.0\%) presented with tandem vessel occlusions involving the ICA or a proximal MCA segment, or proximal occlusion that had moved distally between CTA and angiography. 18 patients received intravenous tPA $(41.9 \%) .42$ cases were treated with a stent-retriever, and 1 case was treated with intra-arterial tPA. Multiple devices were used, however all were $3-4 \mathrm{~mm}$ in diameter, and 39 were $15-20 \mathrm{~mm}$ in length. 3 cases used a $4 \times 40 \mathrm{~mm}$ device. The mean number of passes with a device was 1.2 (range 13). Near or complete reperfusion (mTICI score $2 \mathrm{~b}$ or greater) was achieved in 40 cases $(93.0 \%)$. Median initial NIHSS on presentation was 10.5 (IQR 7.8-18). Median discharge NIHSS was 2 (IQR 1-5.3). Initial and discharge NIHSS was not available in 3 patients. 90-day mRS was $0-2$ in 17 patients (48.6\% of those available) and $3-6$ in 18 patients $(51.4 \%$ of those available). 90-day mRS was not available in 9 patients (18.6\% of total), mostly in the earliest cases found in the database. 3 cases of intraparenchymal hemorrhage occurred (7.0\%), 2 of which were symptomatic including 1 resultant death. 
Conclusion Distal middle cerebral artery thrombectomy with stent-retriever devices is achievable and can be safe and technically effective. Randomized controlled trials have not been performed to determine if thrombectomy in or distal to the M2-3 junction is more beneficial than other techniques, such as aspiration thrombectomy, intravenous thrombolysis, or maximum medical therapy.

Disclosures B. Donegan: None. J. Loeb: None. C. Martin: None. W. Holloway: None. J. Halpin: None. N. Akhtar: None.

\section{E-130 INCIDENCE AND OUTCOMES OF LARGE VESSEL OCCLUSION STROKE AFTER CARDIAC SURGERY AT A MAJOR ACADEMIC MEDICAL CENTER}

'D Wilkinson*, 'S Koduri, ${ }^{2} \mathrm{~J}$ Burke, ${ }^{3} \mathrm{~J}$ Gemmete, ${ }^{3} \mathrm{~N}$ Chaudhary, ${ }^{4} \mathrm{H}$ Patel, ${ }^{1} \mathrm{~A}$ Pandey. ${ }^{1}$ Neurosurgery, University of Michigan, Ann Arbor, Ml; ${ }^{2}$ Neurology, University of Michigan, Ann Arbor, Ml; ${ }^{3}$ Radiology, University of Michigan, Ann Arbor, Ml; ${ }^{4}$ Cardiac Surgery, University of Michigan, Ann Arbor, MI

\subsection{6/neurintsurg-2019-SNIS.205}

Background and purpose Stroke is one of the most feared complications of cardiac surgery. Modern stent-retrieval techniques provide effective treatment for large vessel occlusive strokes. The purpose of this study was to 1) report the incidence of large vessel occlusive stroke after cardiac surgery at a large academic center, and 2) describe outcomes after postoperative large vessel occlusive strokes (LVOs) associated with cardiac surgery.

Methods All patients experiencing stroke within 30 days after undergoing cardiac surgery via an open or endovascular approach at a major academic medical center in 2015-2017 were reviewed. LVOs were identified through review of imaging and medical records and their characteristics and clinical courses were examined.

Results Over the study period, 4,209 cardiac surgeries, including endovascular procedures, were performed. Of 111 patients classified as having stroke, 8 had LVO. Two of the three patients who received mechanical thrombectomy returned to independent living, compared to only 1 of the 5 patients who did not undergo thrombectomy. In the 2 weeks following cardiac surgery, the rate of LVO was estimated at $200 \mathrm{x}$ that of the general population.

Conclusion Of patients undergoing cardiac surgery at an academic medical center, $0.2 \%(95 \%$ CI $0.1-0.4 \%)$ had LVO within 30 days. Cardiac surgery patients and their caregivers should be counseled about the warning signs of stroke, the importance of timely intervention, and how to seek emergency care in the postoperative period.

Disclosures D. Wilkinson: None. S. Koduri: None. J. Burke: None. J. Gemmete: None. N. Chaudhary: None. H. Patel: None. A. Pandey: None.

\section{E-131 OUTCOMES OF MECHANICAL THROMBECTOMY FOR ACUTE ISCHEMIC STROKE IN NATIVE AMERICAN POPULATION: A PRELIMINARY STUDY}

${ }^{1} \mathrm{~A}$ lkram*, 'S Suriya, ${ }^{1} \mathrm{M}$ Farooqui, ${ }^{1} \mathrm{O}$ Owens, ${ }^{1} \mathrm{~A}$ Alvarado Arias, ${ }^{2} \mathrm{D}$ Sorte, ${ }^{3} \mathrm{~A}$ Carlson, ${ }^{4} \mathrm{~S}$ Ortega-Guiterrez, ${ }^{1} \mathrm{M}$ Torbey, ${ }^{1} \mathrm{~A}$ Zafar. ${ }^{1}$ Neurology, University of New Mexico Health Sciences Center, Albuquerque, NM; ${ }^{2}$ Neurourgeny, University of New Mexico Health Sciences Center, Albuquerque, NM; ${ }^{3}$ Neurosurgery, University of New Mexico Health Sciences Center, Albuquerque, NM; ${ }^{4}$ Neurology, University of lowa Hospital, lowa City, IA
Objectives The primary objective of this study is to evaluate the difference in the functional and neurological outcomes after mechanical thrombectomy (MT) for an acute ischemic stroke (AIS), between Native Americans (NA) patients versus other ethnicities.

Background There is scarcity of data regarding outcome of MT in NA population. Considering the dynamics of the state of New Mexico and a diverse patient population, we evaluated the difference in outcome between NA patients with comparison group representing other ethnicities.

Methods This is a preliminary, observational retrospective case-control study. All NA patients who underwent MT for LVO from January 01, 2016 to December 31, 2018 at UNMH were included. Data was extracted from Cerner database at UNMH and was collected on secured Redcap ${ }^{\mathrm{TM}}$ database. Patients were categorized into NA vs comparison group (other ethnicities) into two groups, with the ratio of $1: 4$ respectively.

Results A total of 35 patients who underwent MT at UNMH for an AIS were included in analysis: seven NA and 28 controls. Nine patients $(\mathrm{NA}=7)$ were directly admitted through ED while 26 were transferred from outside hospitals $(\mathrm{NA}=6)$. Prevalence of vascular risk factors, including Hypertension, hyperlipidemia, Coronary artery disease, Diabetes Mellitus was noticeably higher in NA than the comparison group. There was no statistical difference in the two groups with regards to median time interval from last known well (LKW) to groin puncture, and from LKW to reperfusion. The mortality was $14 \%$ in NA group and $4 \%$ in the comparison group. The median NIHSS on discharge between NA and comparison group was 13 and 15.5, respectively. The median mRS at 6 months follow up was 1 in NA and 2 in comparison group, however this was not statistically significant.

Abstract E-131 Table 1 Demographics and comorbidities of patients who underwent Mechanical Thrombectomy (MT)

\begin{tabular}{lll}
\hline & $\begin{array}{l}\text { Natives with MT } \\
(\mathrm{N}=7)\end{array}$ & $\begin{array}{l}\text { Comparison group with MT } \\
(\mathrm{N}=28)\end{array}$ \\
\hline Mean Age & 63 & 69 \\
Gender & $3(43 \%)$ & $13(46 \%)$ \\
- Female & $4(57 \%)$ & $15(53 \%)$ \\
- Male & & \\
Ethnicity & $0(0 \%)$ & $19(68 \%)$ \\
- Caucasian & $0(0 \%)$ & $1(4 \%)$ \\
- African & $0(0 \%)$ & $6(21 \%)$ \\
- Hispanic & $7(100 \%)$ & $0(0 \%)$ \\
- Native American & $0(0 \%)$ & $2(7 \%)$ \\
- Unknown & & \\
OSH Transfers & $6(86 \%)$ & $20(71 \%)$ \\
TPA given & $4(57 \%)$ & $18(62 \%)$ \\
Medical History & $6(86 \%)$ & $13(52 \%)$ \\
- HTN & $5(71 \%)$ & $4(14 \%)$ \\
- HLD & $2(29 \%)$ & $4(14 \%)$ \\
- CHF & $3(43 \%)$ & $2(7 \%)$ \\
- CAD & $0(0 \%)$ & $9(32 \%)$ \\
- AF & $3(43 \%)$ & $6(21 \%)$ \\
- DM & $2(29 \%)$ & $1(4 \%)$ \\
- Previous IS & $0(0 \%)$ & $0(0 \%)$ \\
- Previous HS & $2(29 \%)$ & $9(32 \%)$ \\
- Tobacco use & & \\
\hline & & \\
- & & \\
\hline
\end{tabular}

